\title{
Epidermoid metaplasia of the esophagus with an unusual appearance on magnification
}

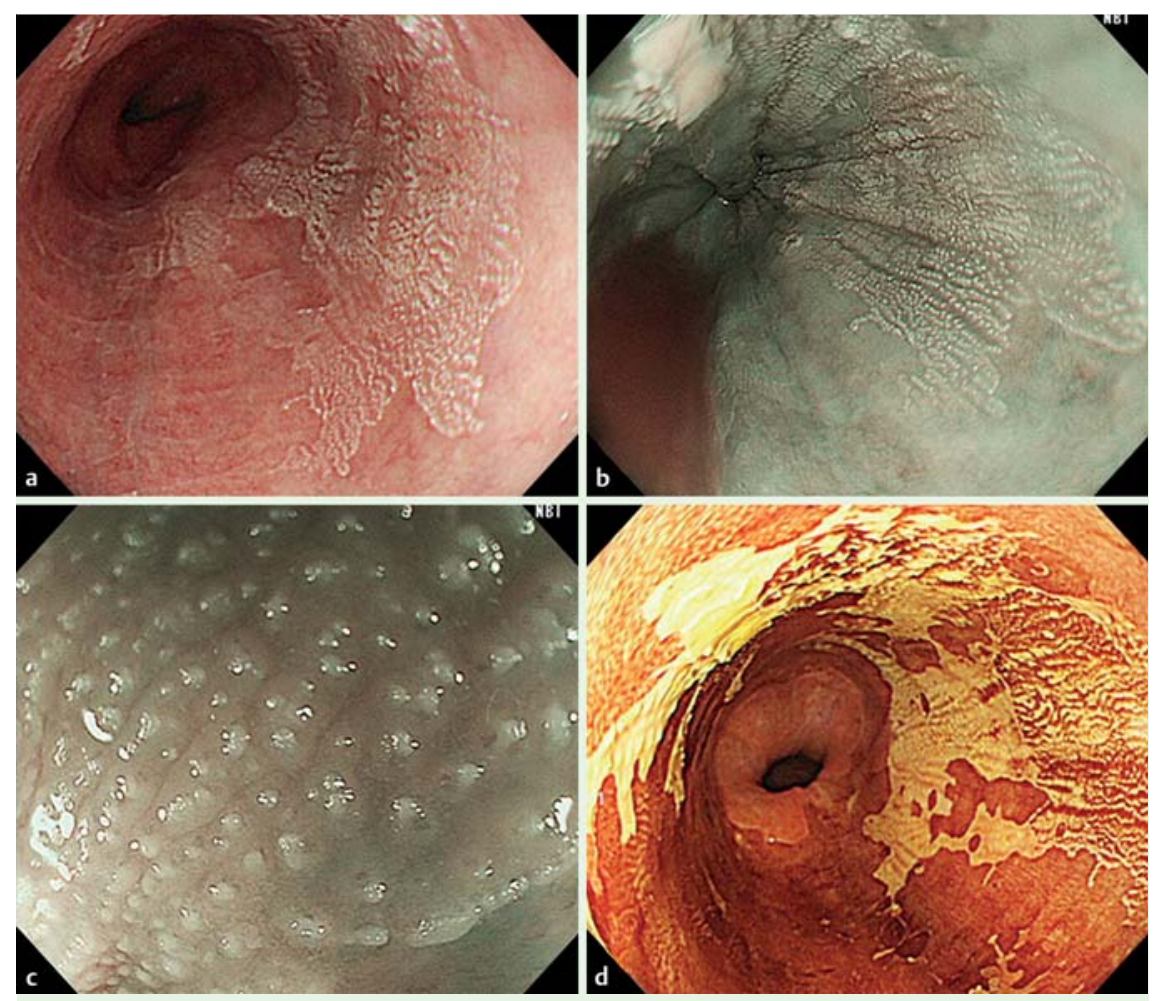

Fig. 1 Endoscopic imaging of esophageal epidermoid metaplasia. a White-light endoscopy reveals white, plaque-like lesions arranged semi-circumferentially around the middle to distal esophagus. b The white plaques have a nodular pattern at lower magnification (narrow-band imaging). c A characteristic appearance, similar to that of goose flesh, is visible at higher magnification (narrow-band imaging). d After iodine staining, a well-demarcated unstained area is seen.

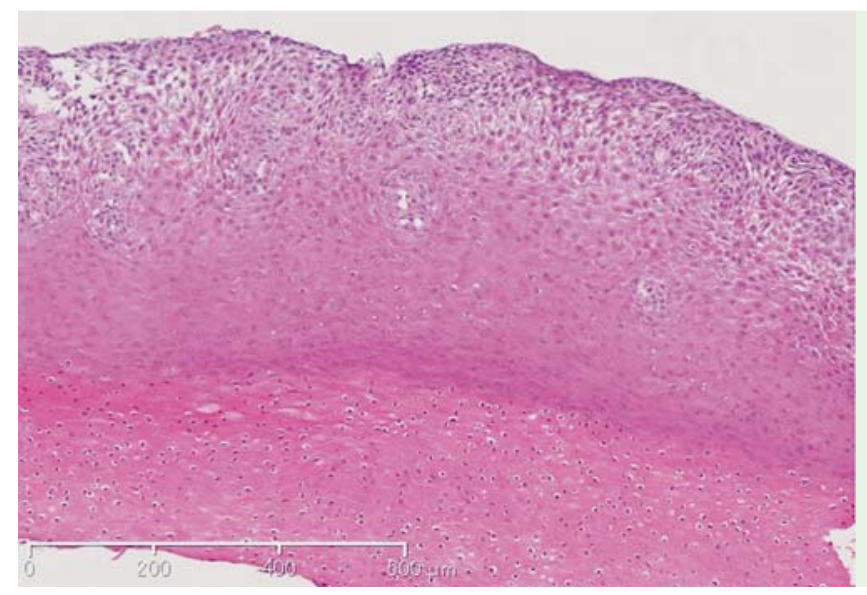

Fig. 2 Microscopic imaging. Hyperkeratotic and granular layers are seen in the esophageal epithelium after hematoxylin and eosin staining of a biopsy specimen section.

A 69-year-old man who had a history of a distal gastrectomy for a hemorrhagic duodenal ulcer 50 years earlier was referred to our hospital with an esophageal lesion. The lesion had been detected by esophagogastroduodenoscopy during health screening. The results of his physical ex- amination were normal. He smoked 20 cigarettes daily and drank alcohol almost every day.

Endoscopy revealed white, plaque-like lesions distributed in a semi-circumferential arrangement from the middle to the distal esophagus ( $\bullet$ Fig.1a). On narrow-band imaging, the white plaques had a nodular pattern at lower magnification ( $\bullet$ Fig. 1 b) and a characteristic appearance, similar to that of goose flesh, at higher magnification $(\diamond$ Fig.1 c). After iodine staining, a welldemarcated unstained area was seen ( Fig.1d). The appearance of biopsy specimens taken from the lesion and stained with hematoxylin and eosin led to a diagnosis of epidermoid metaplasia with hyperkeratotic and granular layers in the epithelium, mimicking the corneal layer of the epidermis ( $\bullet$ Fig.2). No further examination was planned.

Epidermoid metaplasia of the esophagus is a rare condition affecting the middle to distal esophagus in middle-aged to elderly persons [1]. This lesion and several similar lesions, including esophageal hyperkeratosis [2] and papillomatosis [3], are considered to develop as an unusual response to chemical or mechanical irritation, such as acid reflux [4]. One report suggested alcohol abuse to be a possible cause of esophageal epidermal metaplasia, and this lesion has also been suggested to be strongly associated with squamous cell carcinoma (of the esophagus and oropharynx) [5], although the etiology remains unclear.

Because the endoscopic features of epidermoid metaplasia (i.e., slightly elevated shape, translucent white color, scaly or shaggy surface, and clear area after iodine staining) resemble those of superficial esophageal cancer [5], it is recommended that endoscopists pay more attention to this unique lesion. Detailed investigations of larger numbers of patients are required.

Endoscopy_UCTN_Code_CCL_1AB_2AC_3AH

Competing interests: None

\section{Shinji Kitamura', Yasuyuki Okada', Naoki Muguruma', Hiroshi Miyamoto', Yoshimi Bando², Rika Aoki³ Tetsuji Takayama'}

${ }^{1}$ Department of Gastroenterology and Oncology, The University of Tokushima Graduate School, Tokushima, Japan

${ }^{2}$ Division of Pathology, Tokushima University Hospital, Tokushima, Japan 3 Tokushima Health Screening Center, Tokushima, Japan 


\section{References}

1 Singhi $A D$, Arnold $C A$, Crowder $C D$ et al. Esophageal leukoplakia or epidermoid metaplasia: a clinicopathological study of 18 patients. Mod Pathol 2014; 27: 38 - 43

2 Kisloff B, McGrath KM, Davison JM. Esophageal hyperkeratosis in a healthy adult. Clin Gastroenterol Hepatol 2011; 9: A20

3 Brandt LJ. A rare benign esophageal lesion. Gastrointest Endosc 2014; 80: 731 - 732

4 Fukui T, Sakurai T, Miyamoto $S$ et al. Education and imaging. Gastrointestinal: epidermal metaplasia of the esophagus. J Gastroenterol Hepatol 2006; 21: 1627
5 Ezoe $Y$, Fujii S, Muto $M$ et al. Epidermoid metaplasia of the esophagus: endoscopic feature and differential diagnosis. Hepatogastroenterology 2011; 58: 809-813

\section{Bibliography}

Dol http://dx.doi.org/

10.1055/s-0034-1391299

Endoscopy 2015; 47: E100-E101

(c) Georg Thieme Verlag KG

Stuttgart · New York

ISSN 0013-726X

\section{Corresponding author}

\section{Naoki Muguruma, MD, PhD}

Department of Gastroenterology and Oncology The University of Tokushima Graduate School 3-18-15, Kuramoto-cho

Tokushima City, 770-8503

Japan

Fax: +81-88-633-9235

muguruma.clin.med@gmail.com 